\title{
A diagrammatic analysis of two-body charmed baryon decays with flavor symmetry
}

\author{
H.J. Zhao, ${ }^{a}$ Yan-Li Wang, ${ }^{a}$ Y.K. Hsiao ${ }^{a}$ and Yao $\mathbf{Y u}^{b}$ \\ ${ }^{a}$ School of Physics and Information Engineering, Shanxi Normal University, \\ Linfen 041004, China \\ ${ }^{b}$ Chongqing University of Posts $\&$ Telecommunications, \\ Chongqing, 400065, China \\ E-mail: hjzhao@163.com, 1556233556@qq.com, yukuohsiao@gmail.com, \\ yuyao@cqupt.edu.cn
}

ABSTRACT: We study the two-body anti-triplet charmed baryon decays based on the diagrammatic approach with $\mathrm{SU}(3)$ flavor symmetry. We extract the two $W$-exchange effects as $E_{\mathbf{B}}$ and $E^{\prime}$ that contribute to the $\Lambda_{c}^{+} \rightarrow \Xi^{0} K^{+}$decay, together with the relative phases, where $E_{\mathbf{B}}$ gives the main contribution. Besides, we find that $\mathcal{B}\left(\Lambda_{c}^{+} \rightarrow p \pi^{0}\right)=$ $\left(0.8_{-0.8}^{+0.9}\right) \times 10^{-4}$, which is within the experimental upper bound. Particularly, we obtain $\mathcal{B}\left(\Xi_{c}^{+} \rightarrow \Xi^{0} \pi^{+}\right)=(9.3 \pm 3.6) \times 10^{-3}, \mathcal{B}\left(\Xi_{c}^{0} \rightarrow \Xi^{-} \pi^{+}, \Lambda^{0} \bar{K}^{0}\right)=(19.3 \pm 2.8,8.3 \pm 5.0) \times 10^{-2}$ and $\mathcal{B}\left(\Xi_{c}^{0} \rightarrow \Xi^{-} K^{+}\right)=(5.6 \pm 0.8) \times 10^{-4}$, which all agree with the data. For the singly Cabibbo suppressed $\Lambda_{c}^{+}$decays, we predict that $\mathcal{B}\left(\Lambda_{c}^{+} \rightarrow n \pi^{+}, p \eta^{\prime}, \Sigma^{+} K^{0}\right)=(7.7 \pm$ $2.0,7.1 \pm 1.4,19.1 \pm 4.8) \times 10^{-4}$, which are accessible to the experiments at BESIII, BELLEII and $\mathrm{LHCb}$.

Keywords: Branching fraction, Charm physics, Flavor physics, Rare decay, e+-e- Experiments

ArXiv EPrint: 1811.07265 


\section{Contents}

1 Introduction 1

2 Diagrammatic approach $\quad 2$

3 Numerical results 3

4 Discussions and conclusions 5

\section{Introduction}

The experimental studies of two-body $\mathbf{B}_{c} \rightarrow \mathbf{B} M$ decays have provided important information for the theoretical understanding of the hadronization in the weak interaction, where $\mathbf{B}_{c}=\left(\Xi_{c}^{0}, \Xi_{c}^{+}, \Lambda_{c}^{+}\right)$are the lowest-lying anti-triplet charmed baryon states, and $\mathbf{B}(M)$ the baryon (meson) state. For example, the BESIII collaboration has recently measured the purely non-factorizable decays, of which the branching fractions are given by [1]

$$
\begin{aligned}
\mathcal{B}\left(\Lambda_{c}^{+} \rightarrow \Xi^{0} K^{+}\right) & =(5.90 \pm 0.86 \pm 0.39) \times 10^{-3}, \\
\mathcal{B}\left(\Lambda_{c}^{+} \rightarrow \Xi^{* 0} K^{+}\right) & =(5.02 \pm 0.99 \pm 0.31) \times 10^{-3},
\end{aligned}
$$

with $\Xi^{* 0} \equiv \Xi(1530)^{0}$. This implies that the non-factorizable effects can be as significant as the factorizable ones in $\mathbf{B}_{c} \rightarrow \mathbf{B} M[2,3]$, although being often neglected in the b-hadron decays $[4-7]$.

Some theoretical approaches have tried to deal with the non-factorizable effects [8-12]. Nonetheless, $\mathcal{B}\left(\Lambda_{c}^{+} \rightarrow \Xi^{0} K^{+}\right)$is calculated to be 2-6 times smaller than the observation. Without involving the detailed dynamics, the approach based on the $\mathrm{SU}(3)$ flavor $\left(\mathrm{SU}(3)_{f}\right)$ symmetry is able to receive all contributions [13-31], such that $\mathcal{B}\left(\mathbf{B}_{c} \rightarrow \mathbf{B} M\right)$ can be explained [22-25]; particularly, $\mathcal{B}\left(\Lambda_{c}^{+} \rightarrow \Xi^{0} K^{+}\right)$. However, the $\mathrm{SU}(3)_{f}$ symmetry mixes the factorizable and non-factorizable effects, instead of quantifying their individual contributions.

As depicted in figure 1, one can identify the (non-)factoriable effects by the topological diagrams, and parameterize them as the topological amplitudes [32-34]. Accordingly, $\Lambda_{c}^{+} \rightarrow \Xi^{(*) 0} K^{+}$is seen to decay through the two $W$-exchange ones in figures $1(\mathrm{~d}, \mathrm{e})$. Since the topological diagrams have been commonly used in the calculations and measurements $[1,8-12,32,35,36]$, their information can be important. Therefore, we propose to perform the numerical analysis with the topological amplitudes, such that we can determine the sizes and relative phases for the different effects. Note that the same numerical analysis known as the diagrammatic approach has been well performed to extract the topological amplitudes in the $D$ decays [37-42]. In this paper, we will demonstrate that 


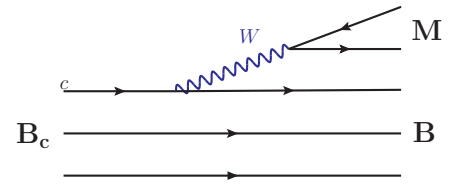

(a) $T$

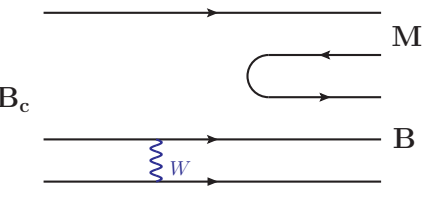

(d) $E^{\prime}$

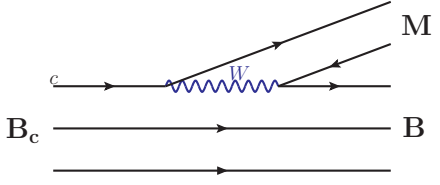

(b) $C$

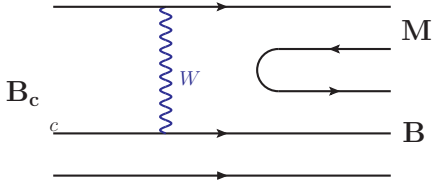

(e) $E_{\mathrm{B}}$

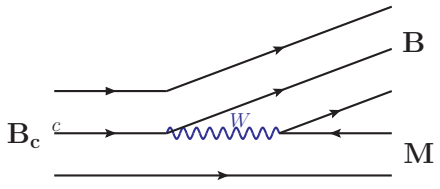

(c) $C^{\prime}$

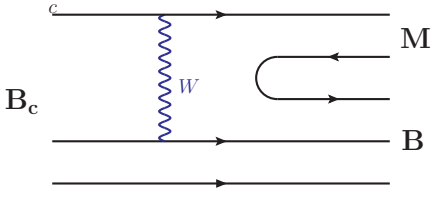

(f) $E_{M}$

Figure 1. Topological diagrams for the $\mathbf{B}_{c} \rightarrow \mathbf{B} M$ decays.

the topological amplitudes can explain the data. Particularly, in our fit we will be able to accommodate the experimental measurements of $\mathcal{B}\left(\Lambda_{c}^{+} \rightarrow p \pi^{0}\right)<2.7 \times 10^{-4}[3]$ and $\mathcal{B}\left(\Xi_{c}^{0} \rightarrow \Xi^{-} K^{+}\right) / \mathcal{B}\left(\Xi_{c}^{0} \rightarrow \Xi^{-} \pi^{+}\right)=(0.56 \pm 0.12) s_{c}^{2}$ [43], where $s_{c}=V_{u s}$. We will also predict the branching fractions for the $\mathbf{B}_{c} \rightarrow \mathbf{B} M$ decays that have not been observed yet.

\section{Diagrammatic approach}

For the two-body charmed baryon decays, the relevant effective Hamiltonian is given by [44]

$$
\mathcal{H}_{\mathrm{eff}}=\sum_{i=1,2} \frac{G_{F}}{\sqrt{2}} c_{i}\left(V_{c s} V_{u d} O_{i}+V_{c q} V_{u q} O_{i}^{q}+V_{c d} V_{u s} O_{i}^{\prime}\right)
$$

with $q=(d, s)$, where $G_{F}$ is the Fermi constant, $c_{1,2}$ are the Wilson coefficients, and $V_{i j}$ the CKM matrix elements. The four-quark operators $O_{1,2}^{(q)}$ and $O_{1,2}^{\prime}$ are written as

$$
\begin{array}{ll}
O_{1}=(\bar{u} d)(\bar{s} c), & O_{2}=(\bar{s} d)(\bar{u} c), \\
O_{1}^{q}=(\bar{u} q)(\bar{q} c), & O_{2}^{q}=(\bar{q} q)(\bar{u} c), \\
O_{1}^{\prime}=(\bar{u} s)(\bar{d} c), & O_{2}^{\prime}=(\bar{d} s)(\bar{s} c),
\end{array}
$$

with $q=(d, s)$ and $\left(\bar{q}_{1} q_{2}\right)=\bar{q}_{1} \gamma_{\mu}\left(1-\gamma_{5}\right) q_{2}$. The decays with $\left|V_{c s} V_{u d}\right| \simeq 1,\left|V_{c q} V_{u q}\right| \simeq s_{c}$ and $\left|V_{c d} V_{u s}\right| \simeq s_{c}^{2}$ are classified as the Cabibbo-favored (CF), singly Cabibbo-suppressed (SCS) and doubly Cabibbo-suppressed (DCS) processes, respectively.

By using $\mathcal{H}_{\text {eff }}$ in eq. (2.1), we draw different topological diagrams in $\mathbf{B}_{c} \rightarrow \mathbf{B} M[32-$ 34], where the quark lines should be in accordance with the operators in eq. (2.2). As seen in figure 1, we obtain six topological diagrams. The external and internal $W$-emission diagrams in figures 1a and $\mathrm{b}$ can be parameterized as the topological amplitudes $T$ and $C$, respectively. Since one can factorize $T$ and $C$ as $\mathcal{A} \propto\left\langle M\left|\left(\bar{q}_{1} q_{2}\right)\right| 0\right\rangle\left\langle\mathbf{B}\left|\left(\bar{q}_{3} c\right)\right| \mathbf{B}_{c}\right\rangle$ [4], which consists of two calculable matrix elements, the $T$ and $C$ are regarded as the factorizable amplitudes $[7,22,32]$. The other internal $W$-emission diagram in figure $1 \mathrm{c}$ has no factorizable 
form, parameterized as $C^{\prime}$. In figures $1(\mathrm{~d}, \mathrm{e}, \mathrm{f})$, the $W$-exchange amplitudes of $\left(E^{\prime}, E_{\mathbf{B}}, E_{M}\right)$ need an additional gluon to relate $M$ and $\mathbf{B}$. Besides, $E_{\mathbf{B}}\left(E_{M}\right)$ has the $W$-boson to connect $\mathbf{B}$ and $M$, with the $c$-quark transition to be a valence quark in $\mathbf{B}(M)$, whereas $M$ in the $E^{\prime}$ amplitude is unable to connect to the $W$-boson. In addition to $C^{\prime},\left(E^{\prime}, E_{\mathbf{B}}, E_{M}\right)$ are the non-factorizable amplitudes according to the factorization approach $[32,35]$. As a result, we clearly identify each (non-)factorizable effect that contribute to the two-body $\mathbf{B}_{c} \rightarrow \mathbf{B} M$ decays.

To present the amplitudes of $\mathbf{B}_{c} \rightarrow \mathbf{B} M$ with $(T, C)$ and $\left(C^{\prime}, E^{\prime}, E_{\mathbf{B}}, E_{M}\right)$, we need the suitable insertions of the final states to match the quark lines, such as $\pi^{0}=\sqrt{1 / 2}(u \bar{u}-d \bar{d})$, which adds a pre-factor of $\pm \sqrt{1 / 2}$ to the topological parameters. Likewise, the $\left(\eta, \eta^{\prime}\right)$ meson states that mix with $\eta_{q}=\sqrt{1 / 2}(u \bar{u}+d \bar{d})$ and $\eta_{s}=s \bar{s}$ lead to the other pre-factors. Specifically, the mixing matrix is presented as [45]

$$
\left(\begin{array}{c}
\eta \\
\eta^{\prime}
\end{array}\right)=\left(\begin{array}{cc}
\cos \phi & -\sin \phi \\
\sin \phi & \cos \phi
\end{array}\right)\left(\begin{array}{l}
\eta_{q} \\
\eta_{s}
\end{array}\right),
$$

with the mixing angle $\phi=(39.3 \pm 1.0)^{\circ}$. We hence obtain the amplitudes of $\mathbf{B}_{c} \rightarrow$ $\mathbf{B} M$, given in table 1. The topological amplitudes are in fact complex, presented as 11 parameters:

$$
T, C e^{i \delta_{C}}, C^{\prime} e^{i \delta_{C^{\prime}}}, E_{\mathbf{B}} e^{i \delta_{E_{\mathbf{B}}}}, E_{M} e^{i \delta_{E_{M}}}, E^{\prime} e^{i \delta_{E^{\prime}}},
$$

with $T$ set to be relatively real. To obtain the decay widths, we depend on the integration of the phase space for the two-body decays, given by [43]

$$
\begin{aligned}
\Gamma\left(\mathbf{B}_{c} \rightarrow \mathbf{B} M\right) & =\frac{\left|\vec{p}_{\mathbf{B}}\right|}{8 \pi m_{\mathbf{B}_{c}}^{2}}\left|\mathcal{A}\left(\mathbf{B}_{c} \rightarrow \mathbf{B} M\right)\right|^{2}, \\
\left|\vec{p}_{\mathbf{B}}\right| & =\frac{\sqrt{\left[m_{\mathbf{B}_{c}}^{2}-\left(m_{\mathbf{B}}+m_{M}\right)^{2}\right]\left[m_{\mathbf{B}_{c}}^{2}-\left(m_{\mathbf{B}}-m_{M}\right)^{2}\right]}}{2 m_{\mathbf{B}_{c}}} .
\end{aligned}
$$

with $\mathcal{A}\left(\mathbf{B}_{c} \rightarrow \mathbf{B} M\right)$ from table 1 .

\section{Numerical results}

In the numerical analysis, we perform a minimum $\chi^{2}$-fit, with the equation written as [25]

$$
\chi^{2}=\sum_{i}\left(\frac{\mathcal{B}_{\mathrm{th}}^{i}-\mathcal{B}_{\mathrm{ex}}^{i}}{\sigma_{\mathrm{ex}}^{i}}\right)^{2}+\sum_{j}\left(\frac{\mathcal{R}_{\mathrm{th}}^{j}-\mathcal{R}_{\mathrm{ex}}^{j}}{\sigma_{\mathrm{ex}}^{j}}\right)^{2},
$$

where $\mathcal{B}(\mathcal{R})$ denotes (the ratios of) the branching ratios. The subscripts $t h$ and $e x$ stand for the theoretical inputs from the amplitudes in table 1 and the experimental data in table 2 , respectively, with $\sigma_{e x}^{i, j}$ the experimental uncertainties. By putting the recent observation of $\mathcal{B}\left(\Xi_{c}^{0} \rightarrow \Xi^{-} \pi^{+}\right)=(1.80 \pm 0.55) \times 10^{-2}[46]$ into $\mathcal{R}_{1,2}\left(\Xi_{c}^{0}\right)$ in table 2, we determine that $\mathcal{B}\left(\Xi_{c}^{0} \rightarrow \Xi^{-} K^{+}\right)=(5.0 \pm 1.9) \times 10^{-4}$ and $\mathcal{B}\left(\Xi_{c}^{0} \rightarrow \Lambda^{0} \bar{K}^{0}\right)=(7.6 \pm 2.6) \times 10^{-3}$. The $\mathcal{B}\left(\Xi_{c}^{+} \rightarrow\right.$ $\left.\Xi^{0} \pi^{+}\right)$is extracted from the ratio of $\mathcal{B}\left(\Xi_{c}^{+} \rightarrow \Xi^{0} \pi^{+}\right) / \mathcal{B}\left(\Xi_{c}^{+} \rightarrow \Xi^{-} \pi^{+} \pi^{+}\right)=0.55 \pm 0.16[43]$ 


\begin{tabular}{|l|l|}
\hline Decay modes & Amplitudes $/\left(\frac{G_{F}}{\sqrt{2}}\right)$ \\
\hline$\Xi_{c}^{0} \rightarrow \Sigma^{+} K^{-}$ & $V_{c s} V_{u d}\left(E_{M}+E^{\prime}\right)$ \\
$\Xi_{c}^{0} \rightarrow \Sigma(\Lambda)^{0} \bar{K}^{0}$ & $V_{c s} V_{u d}\left(C+C^{\prime}+E_{M}+E^{\prime}\right)$ \\
$\Xi_{c}^{0} \rightarrow \Xi^{0} \pi^{0}$ & $V_{c s} V_{u d} \frac{1}{\sqrt{2}}\left(E_{\mathbf{B}}-C^{\prime}\right)$ \\
$\Xi_{c}^{0} \rightarrow \Xi^{0} \eta$ & $V_{c s} V_{u d}\left[\frac{1}{\sqrt{2}}\left(C^{\prime}+E_{\mathbf{B}}\right) c \phi-\left(E_{M}+E^{\prime}\right) s \phi\right]$ \\
$\Xi_{c}^{0} \rightarrow \Xi^{0} \eta^{\prime}$ & $V_{c s} V_{u d}\left[\frac{1}{\sqrt{2}}\left(C^{\prime}+E_{\mathbf{B}}\right) s \phi+\left(E_{M}+E^{\prime}\right) c \phi\right]$ \\
$\Xi_{c}^{0} \rightarrow \Xi^{-} \pi^{+}$ & $V_{c s} V_{u d}\left(T+E_{\mathbf{B}}\right)$ \\
\hline$\Xi_{c}^{0} \rightarrow \Sigma^{+} \pi^{-}$ & $\lambda_{d} E_{M}+\lambda_{s} E^{\prime}$ \\
$\Xi_{c}^{0} \rightarrow \Sigma^{-} \pi^{+}$ & $\lambda_{d}\left(T+E_{\mathbf{B}}\right)$ \\
$\Xi_{c}^{0} \rightarrow \Sigma(\Lambda)^{0} \pi^{0}$ & $\frac{1}{\sqrt{2}}\left[\lambda_{d}\left(-C-C^{\prime}-E_{M}+E_{\mathbf{B}}\right)+\lambda_{s}\left(E_{\mathbf{B}}-E^{\prime}\right)\right]$ \\
$\Xi_{c}^{0} \rightarrow \Sigma(\Lambda)^{0} \eta$ & $\frac{1}{\sqrt{2}}\left[\lambda_{d}\left(C+C^{\prime}+E_{M}+E_{\mathbf{B}}\right)+\lambda_{s}\left(E_{\mathbf{B}}+E^{\prime}\right)\right] c \phi$ \\
& $-\left[\lambda_{d} E^{\prime}+\lambda_{s}\left(C+C^{\prime}+E_{M}\right)\right] s \phi$ \\
$\Xi_{c}^{0} \rightarrow \Sigma(\Lambda)^{0} \eta^{\prime}$ & $\frac{1}{\sqrt{2}}\left[\lambda_{d}\left(C+C^{\prime}+E_{\mathbf{B}}+E_{M}\right)+\lambda_{s}\left(E_{\mathbf{B}}+E^{\prime}\right)\right] s \phi$ \\
& $+\left[\lambda_{d} E^{\prime}+\lambda_{s}\left(C+C^{\prime}+E_{M}\right)\right] c \phi$ \\
$\Xi_{c}^{0} \rightarrow \Xi^{-} K^{+}$ & $\lambda_{d} E_{\mathbf{B}}+\lambda_{s}\left(T+E_{\mathbf{B}}\right)$ \\
$\Xi_{c}^{0} \rightarrow \Xi^{0} K^{0}$ & $\lambda_{d} E_{M}+\lambda_{s}\left(C^{\prime}+E^{\prime}\right)$ \\
$\Xi_{c}^{0} \rightarrow p K^{-}$ & $\lambda_{d} E^{\prime}+\lambda_{s} E_{M}$ \\
$\Xi_{c}^{0} \rightarrow n \bar{K}^{0}$ & $\lambda_{d}\left(C^{\prime}+E^{\prime}\right)+\lambda_{s} E_{M}$ \\
\hline$\Xi_{c}^{0} \rightarrow p \pi^{-}$ & $V_{c d} V_{u s}\left(E_{M}+E^{\prime}\right)$ \\
$\Xi_{c}^{0} \rightarrow \Sigma^{-} K^{+}$ & $V_{c d} V_{u s}\left(T+E_{\mathbf{B}}\right)$ \\
$\Xi_{c}^{0} \rightarrow \Sigma(\Lambda)^{0} K^{0}$ & $V_{c d} V_{u s}\left(C+C^{\prime}+E_{M}+E^{\prime}\right)$ \\
$\Xi_{c}^{0} \rightarrow n \pi^{0}$ & $V_{c d} V_{u s} \frac{1}{\sqrt{2}}\left(E_{\mathbf{B}}-E_{M}-E^{\prime}\right)$ \\
$\Xi_{c}^{0} \rightarrow n \eta$ & $V_{c d} V_{u s} \frac{1}{\sqrt{2}}\left(E_{\mathbf{B}}+E_{M}+E^{\prime}\right) c \phi-V_{c d} V_{u s} C^{\prime} s \phi$ \\
$\Xi_{c}^{0} \rightarrow n \eta^{\prime}$ & $V_{c d} V_{u s} \frac{1}{\sqrt{2}}\left(E_{\mathbf{B}}+E_{M}+E^{\prime}\right) s \phi+V_{c d} V_{u s} C^{\prime} c \phi$ \\
& \\
& \\
& \\
& \\
& \\
&
\end{tabular}

\begin{tabular}{|l|l|}
\hline Decay modes & Amplitudes $/\left(\frac{G_{F}}{\sqrt{2}}\right)$ \\
\hline$\Xi_{c}^{+} \rightarrow \Sigma^{+} \bar{K}^{0}$ & $V_{c s} V_{u d}\left(C+C^{\prime}\right)$ \\
$\Xi_{c}^{+} \rightarrow \Xi^{0} \pi^{+}$ & $V_{c s} V_{u d}\left(T+C^{\prime}\right)$ \\
\hline$\Xi_{c}^{+} \rightarrow \Sigma(\Lambda)^{0} \pi^{+}$ & $\lambda_{s}\left(E_{\mathbf{B}}+E^{\prime}\right)+\lambda_{d}\left(T+C^{\prime}\right)$ \\
$\Xi_{c}^{+} \rightarrow \Sigma^{+} \pi^{0}$ & $\frac{1}{\sqrt{2}}\left[\lambda_{s}\left(E_{\mathbf{B}}+E^{\prime}\right)-\lambda_{d} C\right]$ \\
$\Xi_{c}^{+} \rightarrow \Sigma^{+} \eta$ & $\frac{1}{\sqrt{2}}\left[\lambda_{d} C+\lambda_{s}\left(E_{\mathbf{B}}+E^{\prime}\right)\right] c \phi-\lambda_{s}\left(C+C^{\prime}+E_{M}\right) s \phi$ \\
$\Xi_{c}^{+} \rightarrow \Sigma^{+} \eta^{\prime}$ & $\frac{1}{\sqrt{2}}\left[\lambda_{d} C+\lambda_{s}\left(E_{\mathbf{B}}+E^{\prime}\right)\right] s \phi+\lambda_{s}\left(C+C^{\prime}+E_{M}\right) c \phi$ \\
$\Xi_{c}^{+} \rightarrow \Xi^{0} K^{+}$ & $\lambda_{s}\left(T+C^{\prime}+E_{\mathbf{B}}+E^{\prime}\right)$ \\
$\Xi_{c}^{+} \rightarrow p \bar{K}^{0}$ & $\lambda_{d} C^{\prime}+\lambda_{s} E_{M}$ \\
\hline$\Xi_{c}^{+} \rightarrow \Sigma(\Lambda)^{0} K^{+}$ & $V_{c d} V_{u s}\left(T+C^{\prime}+E_{\mathbf{B}}+E^{\prime}\right)$ \\
$\Xi_{c}^{+} \rightarrow \Sigma^{+} K^{0}$ & $V_{c d} V_{u s}\left(C+E_{M}\right)$ \\
$\Xi_{c}^{+} \rightarrow p \pi^{0}$ & $V_{c d} V_{u s} \frac{1}{\sqrt{2}}\left(E_{\mathbf{B}}+E^{\prime}-E_{M}\right)$ \\
$\Xi_{c}^{+} \rightarrow p \eta$ & $V_{c d} V_{u s} \frac{1}{\sqrt{2}}\left(E_{\mathbf{B}}+E_{M}+E^{\prime}\right) c \phi-V_{c d} V_{u s} C^{\prime} s \phi$ \\
$\Xi_{c}^{+} \rightarrow p \eta^{\prime}$ & $V_{c d} V_{u s} \frac{1}{\sqrt{2}}\left(E_{\mathbf{B}}+E_{M}+E^{\prime}\right) s \phi+V_{c d} V_{u s} C^{\prime} c \phi$ \\
$\Xi_{c}^{+} \rightarrow n \pi^{+}$ & $V_{c d} V_{u s}\left(E_{\mathbf{B}}+E^{\prime}\right)$ \\
\hline
\end{tabular}

\begin{tabular}{|l|l|}
\hline Decay modes & Amplitudes $/\left(\frac{G_{F}}{\sqrt{2}}\right)$ \\
\hline$\Lambda_{c}^{+} \rightarrow \Sigma(\Lambda)^{0} \pi^{+}$ & $V_{c s} V_{u d}\left(T+C^{\prime}+E_{\mathbf{B}}+E^{\prime}\right)$ \\
$\Lambda_{c}^{+} \rightarrow \Sigma^{+} \pi^{0}$ & $V_{c s} V_{u d} \frac{1}{\sqrt{2}}\left(-C^{\prime}+E_{\mathbf{B}}+E^{\prime}\right)$ \\
$\Lambda_{c}^{+} \rightarrow \Sigma^{+} \eta$ & $V_{c s} V_{u d}\left[\frac{1}{\sqrt{2}}\left(C^{\prime}+E_{\mathbf{B}}+E^{\prime}\right) c \phi-E_{M} s \phi\right]$ \\
$\Lambda_{c}^{+} \rightarrow \Sigma^{+} \eta^{\prime}$ & $V_{c s} V_{u d}\left[\frac{1}{\sqrt{2}}\left(C^{\prime}+E_{\mathbf{B}}+E^{\prime}\right) s \phi+E_{M} c \phi\right]$ \\
$\Lambda_{c}^{+} \rightarrow \Xi^{0} K^{+}$ & $V_{c s} V_{u d}\left(E_{\mathbf{B}}+E^{\prime}\right)$ \\
$\Lambda_{c}^{+} \rightarrow p \bar{K}^{0}$ & $V_{c s} V_{u d}\left(C+E_{M}\right)$ \\
\hline$\Lambda_{c}^{+} \rightarrow \Sigma^{+} K^{0}$ & $\lambda_{d} E_{M}+\lambda_{s} C^{\prime}$ \\
$\Lambda_{c}^{+} \rightarrow \Sigma(\Lambda)^{0} K^{+}$ & $\lambda_{d}\left(E_{\mathbf{B}}+E^{\prime}\right)+\lambda_{s}\left(T+C^{\prime}\right)$ \\
$\Lambda_{c}^{+} \rightarrow p \pi^{0}$ & $\lambda_{d} \frac{1}{\sqrt{2}}\left(-C-C^{\prime}-E_{M}+E_{\mathbf{B}}+E^{\prime}\right)$ \\
$\Lambda_{c}^{+} \rightarrow p \eta$ & $\lambda_{d} \frac{1}{\sqrt{2}}\left(C+C^{\prime}+E_{M}+E_{\mathbf{B}}+E^{\prime}\right) c \phi-\lambda_{s} C s \phi$ \\
$\Lambda_{c}^{+} \rightarrow p \eta^{\prime}$ & $\lambda_{d} \frac{1}{\sqrt{2}}\left(C+C^{\prime}+E_{M}+E_{\mathbf{B}}+E^{\prime}\right) s \phi+\lambda_{s} C c \phi$ \\
$\Lambda_{c}^{+} \rightarrow n \pi^{+}$ & $\lambda_{d}\left(T+C^{\prime}+E_{\mathbf{B}}+E^{\prime}\right)$ \\
\hline$\Lambda_{c}^{+} \rightarrow p K^{0}$ & $V_{c d} V_{u s}\left(C+C^{\prime}\right)$ \\
$\Lambda_{c}^{+} \rightarrow n K^{+}$ & $V_{c d} V_{u s}\left(T+C^{\prime}\right)$ \\
\hline
\end{tabular}

Table 1. Amplitudes of $\mathbf{B}_{c} \rightarrow \mathbf{B} M$, where $\lambda_{q} \equiv V_{c q} V_{u q}$ with $q=(d, s)$ and $(s \phi, c \phi) \equiv(\sin \phi, \cos \phi)$ for the $\eta-\eta^{\prime}$ mixing.

and the newly observed $\mathcal{B}\left(\Xi_{c}^{+} \rightarrow \Xi^{-} \pi^{+} \pi^{+}\right)=(2.86 \pm 1.21 \pm 0.38) \times 10^{-2}$ [49]. We adopt the CKM matrix elements in the Wolfenstein parameterization, given by [43]

$$
\left(V_{c s}, V_{u d}, V_{u s}, V_{c d}\right)=\left(1-\lambda^{2} / 2,1-\lambda^{2} / 2, \lambda,-\lambda\right),
$$

with $\lambda=s_{c}=0.22453 \pm 0.00044$. Subsequently, we fit that

$$
\begin{aligned}
\left(T, C, C^{\prime}\right) & =(0.41 \pm 0.02,0.47 \pm 0.08,0.25 \pm 0.02) \mathrm{GeV}^{3} \\
\left(E_{\mathbf{B}}, E_{M}, E^{\prime}\right) & =(0.43 \pm 0.04,0.14 \pm 0.03,0.14 \pm 0.07) \mathrm{GeV}^{3}, \\
\left(\delta_{C}, \delta_{C^{\prime}}, \delta_{E_{\mathbf{B}}}, \delta_{E_{M}}, \delta_{E^{\prime}}\right) & =(31.3 \pm 9.6,158.1 \pm 5.3,-81.0 \pm 8.8,-32.8 \pm 21.6,-88.0 \pm 1.9)^{\circ}, \\
\chi^{2} / \text { n.d.f } & =0.5
\end{aligned}
$$




\begin{tabular}{|c|c|}
\hline Branching ratios & Data \\
\hline $10^{2} \mathcal{B}\left(\Lambda_{c}^{+} \rightarrow p \bar{K}^{0}\right)$ & $3.16 \pm 0.16[43]$ \\
$10^{2} \mathcal{B}\left(\Lambda_{c}^{+} \rightarrow \Lambda^{0} \pi^{+}\right)$ & $1.30 \pm 0.07[43]$ \\
$10^{2} \mathcal{B}\left(\Lambda_{c}^{+} \rightarrow \Sigma^{0} \pi^{+}\right)$ & $1.29 \pm 0.07[43]$ \\
$10^{2} \mathcal{B}\left(\Lambda_{c}^{+} \rightarrow \Sigma^{+} \pi^{0}\right)$ & $1.24 \pm 0.10[43]$ \\
$10^{2} \mathcal{B}\left(\Lambda_{c}^{+} \rightarrow \Xi^{0} K^{+}\right)$ & $0.59 \pm 0.09[1]$ \\
$10^{2} \mathcal{B}\left(\Lambda_{c}^{+} \rightarrow \Sigma^{+} \eta\right)$ & $0.41 \pm 0.20[47]$ \\
$10^{2} \mathcal{B}\left(\Lambda_{c}^{+} \rightarrow \Sigma^{+} \eta^{\prime}\right)$ & $1.34 \pm 0.57[47]$ \\
& \\
\hline
\end{tabular}

\begin{tabular}{|c|c|}
\hline (Ratios of) Branching ratios & Data \\
\hline $10^{4} \mathcal{B}\left(\Lambda_{c}^{+} \rightarrow p \pi^{0}\right)$ & $0.8 \pm 1.4(<0.27)[3,48]$ \\
$10^{4} \mathcal{B}\left(\Lambda_{c}^{+} \rightarrow \Lambda^{0} K^{+}\right)$ & $6.1 \pm 1.2[43]$ \\
$10^{4} \mathcal{B}\left(\Lambda_{c}^{+} \rightarrow \Sigma^{0} K^{+}\right)$ & $5.2 \pm 0.8[43]$ \\
$10^{4} \mathcal{B}\left(\Lambda_{c}^{+} \rightarrow p \eta\right)$ & $12.4 \pm 3.0[43]$ \\
$10^{2} \mathcal{B}\left(\Xi_{c}^{0} \rightarrow \Xi^{-} \pi^{+}\right)$ & $1.80 \pm 0.55[46]$ \\
$\mathcal{R}_{1}\left(\Xi_{c}^{0}\right) \equiv \frac{\mathcal{B}\left(\Xi_{c}^{0} \rightarrow \Xi^{-} K^{+}\right)}{\mathcal{B}\left(\Xi_{c}^{0} \rightarrow \Xi^{-} \pi^{+}\right)}$ & $(0.56 \pm 0.12) s_{c}^{2}[43]$ \\
$\mathcal{R}_{2}\left(\Xi_{c}^{0}\right) \equiv \frac{\mathcal{B}\left(\Xi_{c}^{0} \rightarrow \Lambda^{0} \bar{K}^{0}\right)}{\mathcal{B}\left(\Xi_{c}^{0} \rightarrow \Xi^{-} \pi^{+}\right)}$ & $0.42 \pm 0.06[43]$ \\
$10^{2} \mathcal{B}\left(\Xi_{c}^{+} \rightarrow \Xi^{0} \pi^{+}\right)$ & $1.57 \pm 0.84[43,49]$ \\
\hline
\end{tabular}

Table 2. The data for $\mathbf{B}_{c} \rightarrow \mathbf{B} M$.

with $n . d . f=4$ as the number of degrees of freedom, by which we present the branching ratios of the $\mathbf{B}_{c} \rightarrow \mathbf{B} M$ decays in table 3, together with the recent theoretical results for comparison.

\section{Discussions and conclusions}

Since $\chi^{2} /$ n.d.f $=0.5$ presents a good fit, we demonstrate that the diagrammatic approach can explain the data well. Moreover, the non-factorizable effects depicted in figure 1 now have clear information. The fit of $\left|E_{B}\right| \simeq(|T|,|C|) \simeq 0.4$ and $\left|C^{\prime}\right| \simeq 2\left(\left|E_{M}\right|,\left|E^{\prime}\right|\right) \simeq 0.3$ shows that the non-factorizable effects can be as significant as the factorizable ones; nonetheless, neglected in the factorization approach. While the theoretical computations are unable to explain $\mathcal{B}\left(\Lambda_{c}^{+} \rightarrow \Xi^{0} K^{+}\right.$) [8-12], we explicitly present the two $W$-exchange effects as $E_{\mathbf{B}}$ and $E^{\prime}$ that contribute to $\Lambda_{c}^{+} \rightarrow \Xi^{0} K^{+}$, together with the relative phases. Besides, we point out that $E_{\mathbf{B}}$ has the main contribution. The $E_{M}$ term as the rarely studied $W$-exchange process is found to have a significant interference with $C$ in $\Lambda_{c}^{+} \rightarrow p \bar{K}^{0}$. In contrast, the $\mathrm{SU}(3)_{f}$ parameters cannot distinguish the three $W$-exchange contributions. As seen in table 1 , the $\Lambda_{c}^{+} \rightarrow \Sigma^{+} \eta^{(\prime)}, \Sigma^{+} K^{0}$ decays only receive the non-factorizable effects. Particularly, $C^{\prime}$ and $E_{M}$ in $\Lambda_{c}^{+} \rightarrow \Sigma^{+} K^{0}$ give a constructive interference, leading to $\mathcal{B}\left(\Lambda_{c}^{+} \rightarrow \Sigma^{+} K^{0}\right)=(19.1 \pm 4.8) \times 10^{-4}$ accessible to the BESIII experiment. We hence have a better understanding for the non-factorizable effects.

In the $\mathrm{SU}(3)_{f}$ symmetry, the $\mathcal{B}\left(\Lambda_{c}^{+} \rightarrow p \pi^{0}\right)$ was once overestimated as two times larger than the experimental upper bound [22]. By recovering one of the previously neglected parameters, which gives the destructive interference, the number has been reduced to agree with the data $[30,31]$. It is interesting to note that the recovered parameter is recognized as a factorizable effect, which corresponds to our $C$ term in $\Lambda_{c}^{+} \rightarrow p \pi^{0}$. The $\mathrm{SU}(3)_{f}$ symmetry derives that $\mathcal{A}\left(\Xi_{c}^{0} \rightarrow \Xi^{-} \pi^{+}\right)=s_{c} \mathcal{A}\left(\Xi_{c}^{0} \rightarrow \Xi^{-} K^{+}\right)[22,23,50]$. This causes $\mathcal{R}_{1}\left(\Xi_{c}^{0}\right) \equiv \mathcal{B}\left(\Xi_{c}^{0} \rightarrow \Xi^{-} K^{+}\right) / \mathcal{B}\left(\Xi_{c}^{0} \rightarrow \Xi^{-} \pi^{+}\right) \simeq 1.0 s_{c}^{2}$ to be $4 \sigma$ away from the observation. According to the topological diagrams in figure 2, we find that

$$
\begin{aligned}
& \overline{\mathcal{A}}_{K} \equiv \mathcal{A}\left(\Xi_{c}^{+} \rightarrow \Xi^{-} K^{+}\right) /\left(\frac{G_{F}}{\sqrt{2}}\right)=V_{c s} V_{u s} T+V_{c s} V_{u s} E_{\mathbf{B}}+V_{c d} V_{u d} E_{\mathbf{B}}, \\
& \overline{\mathcal{A}}_{\pi} \equiv \mathcal{A}\left(\Xi_{c}^{+} \rightarrow \Xi^{-} \pi^{+}\right) /\left(\frac{G_{F}}{\sqrt{2}}\right)=V_{c s} V_{u d}\left(T+E_{\mathbf{B}}\right),
\end{aligned}
$$




\begin{tabular}{|c|c|c|c|c|}
\hline$\Xi_{c}^{0}$ & $\mathrm{SU}(3)_{f}$ & Cheng et al. & Our work & Expt. \\
\hline $10^{3} \mathcal{B}_{\Sigma^{+} K^{-}}$ & $7.6 \pm 1.4$ & 7.8 & $22.0 \pm 5.7$ & \\
\hline $10^{3} \mathcal{B}_{\Sigma^{0} \bar{K}^{0}}$ & $0.9_{-0.9}^{+1.1}$ & 0.4 & $7.9 \pm 4.8$ & \\
\hline $10^{3} \mathcal{B}_{\Xi^{0} \pi^{0}}$ & $10.0 \pm 1.4$ & 18.2 & $4.7 \pm 0.9$ & \\
\hline $10^{3} \mathcal{B}_{\Xi^{0} \eta}$ & $13.0 \pm 2.3$ & 26.7 & $8.3 \pm 2.3$ & \\
\hline $10^{3} \mathcal{B}_{\Xi^{0} \eta^{\prime}}$ & $9.1 \pm 4.1$ & & $7.2 \pm 1.9$ & \\
\hline $10^{3} \mathcal{B}_{\Xi^{-} \pi^{+}}$ & $29.5 \pm 1.4$ & 64.7 & $19.3 \pm 2.8$ & $18.0 \pm 5.5$ \\
\hline $10^{3} \mathcal{B}_{\Lambda^{0} \bar{K}^{0}}$ & $14.2 \pm 0.09$ & 13.3 & $8.3 \pm 5.0$ & $7.6 \pm 2.6$ \\
\hline $10^{4} \mathcal{B}_{\Sigma^{+} \pi^{-}}$ & $4.9 \pm 0.9$ & 7.1 & $2.4 \pm 1.5$ & \multirow{12}{*}{$5.0 \pm 1.9$} \\
\hline $10^{4} \mathcal{B}_{\Sigma^{-} \pi^{+}}$ & $18.3 \pm 0.9$ & 26.2 & $11.1 \pm 1.6$ & \\
\hline $10^{4} \mathcal{B}_{\Sigma^{0} \pi^{0}}$ & $5.0 \pm 0.9$ & 3.8 & $1.0 \pm 0.5$ & \\
\hline $10^{4} \mathcal{B}_{\Sigma^{0} \eta}$ & $1.8 \pm 1.1$ & 0.5 & $2.5 \pm 1.3$ & \\
\hline $10^{4} \mathcal{B}_{\Sigma^{0} \eta^{\prime}}$ & $3.2 \pm 2.2$ & & $0.1 \pm 0.1$ & \\
\hline $10^{4} \mathcal{B}_{\Xi^{-} K^{+}}$ & $12.8 \pm 0.6$ & 39.0 & $5.6 \pm 0.8$ & \\
\hline $10^{4} \mathcal{B}_{\Xi^{0} K^{0}}$ & $9.6 \pm 0.4$ & 13.2 & $6.3 \pm 1.9$ & \\
\hline $10^{4} \mathcal{B}_{p K^{-}}$ & $6.0 \pm 1.3$ & 3.5 & $2.5 \pm 1.6$ & \\
\hline $10^{4} \mathcal{B}_{n \bar{K}^{0}}$ & $10.7 \pm 0.6$ & 14.0 & $7.8 \pm 2.3$ & \\
\hline $10^{4} \mathcal{B}_{\Lambda^{0} \pi^{0}}$ & $3.1 \pm 1.1$ & 2.4 & $1.0 \pm 0.5$ & \\
\hline $10^{4} \mathcal{B}_{\Lambda^{0} \eta}$ & $7.9 \pm 2.7$ & 7.7 & $2.6 \pm 1.3$ & \\
\hline $10^{4} \mathcal{B}_{\Lambda^{0} \eta^{\prime}}$ & $16.4 \pm 10.6$ & & $0.2 \pm 0.1$ & \\
\hline $10^{5} \mathcal{B}_{p \pi^{-}}$ & $3.1 \pm 0.7$ & & $7.6 \pm 2.0$ & \\
\hline $10^{5} \mathcal{B}_{\Sigma^{-} K^{+}}$ & $6.1 \pm 0.4$ & & $5.5 \pm 0.7$ & \\
\hline $10^{5} \mathcal{B}_{\Sigma^{0} K^{0}}$ & $2.5 \pm 0.2$ & & $2.3 \pm 1.4$ & \\
\hline $10^{5} \mathcal{B}_{n \pi^{0}}$ & $1.5 \pm 0.4$ & & $3.3 \pm 0.9$ & \\
\hline $10^{5} \mathcal{B}_{n \eta}$ & $5.2 \pm 2.1$ & & $4.2 \pm 0.8$ & \\
\hline $10^{5} \mathcal{B}_{n \eta^{\prime}}$ & $10.2 \pm 7.1$ & & $1.2 \pm 0.3$ & \\
\hline $10^{5} \mathcal{B}_{\Lambda^{0} K^{0}}$ & $0.6 \pm 0.2$ & & $2.4 \pm 1.4$ & \\
\hline
\end{tabular}

\begin{tabular}{|c|ccc|c|}
\hline$\Xi_{c}^{+}$ & $\mathrm{SU}(3)_{f}$ & Cheng et al. & Our work & Expt. \\
\hline $10^{3} \mathcal{B}_{\Sigma^{+} \bar{K}^{0}}$ & $7.8_{-7.8}^{+10.2}$ & 2.0 & $24.1 \pm 7.1$ & \\
$10^{3} \mathcal{B}_{\Xi^{0} \pi^{+}}$ & $4.2 \pm 1.7$ & 17.2 & $9.3 \pm 3.6$ & $15.7 \pm 8.4$ \\
\hline $10^{4} \mathcal{B}_{\Sigma^{0} \pi^{+}}$ & $26.5 \pm 2.5$ & 43.0 & $13.4 \pm 4.9$ & \\
$10^{4} \mathcal{B}_{\Sigma^{+} \pi^{0}}$ & $26.1 \pm 6.7$ & 13.6 & $16.4 \pm 3.2$ & \\
$10^{4} \mathcal{B}_{\Sigma^{+} \eta}$ & $15.0 \pm 10.6$ & 3.2 & $14.1 \pm 3.9$ & \\
$10^{4} \mathcal{B}_{\Sigma^{+} \eta^{\prime}}$ & $34.6 \pm 21.9$ & & $8.7 \pm 3.7$ & \\
$10^{4} \mathcal{B}_{\Xi^{0} K^{+}}$ & $7.6 \pm 1.6$ & 22.0 & $12.3 \pm 3.1$ & \\
$10^{4} \mathcal{B}_{p \bar{K}^{0}}$ & $46.4 \pm 7.2$ & 39.6 & $48.6 \pm 12.2$ & \\
$10^{4} \mathcal{B}_{\Lambda^{0} \pi^{+}}$ & $12.3 \pm 4.2$ & 8.5 & $13.9 \pm 5.1$ & \\
\hline $10^{5} \mathcal{B}_{\Sigma^{0} K^{+}}$ & $11.9 \pm 0.7$ & & $7.2 \pm 1.8$ & \\
$10^{5} \mathcal{B}_{\Sigma^{+} K^{0}}$ & $19.5 \pm 1.7$ & & $16.9 \pm 5.4$ & \\
$10^{5} \mathcal{B}_{p \pi^{0}}$ & $6.0 \pm 1.4$ & & $1.5 \pm 1.5$ & \\
$10^{5} \mathcal{B}_{p \eta}$ & $20.4 \pm 8.4$ & & $16.6 \pm 3.1$ & \\
$10^{5} \mathcal{B}_{p \eta^{\prime}}$ & $40.1 \pm 27.7$ & & $5.8 \pm 1.5$ & \\
$10^{5} \mathcal{B}_{n \pi^{+}}$ & $12.1 \pm 2.8$ & & $5.2 \pm 1.5$ & \\
$10^{5} \mathcal{B}_{\Lambda^{0} K^{+}}$ & $3.3 \pm 0.8$ & & $7.5 \pm 1.9$ & \\
\hline \multicolumn{2}{|r|}{} & & &
\end{tabular}

\begin{tabular}{|c|ccc|c|}
\hline$\Lambda_{c}^{+}$ & $\mathrm{SU}(3)_{f}$ & Cheng et al. & Our work & Expt. \\
\hline $10^{2} \mathcal{B}_{\Sigma^{0} \pi^{+}}$ & $1.26 \pm 0.06$ & 2.24 & $1.26 \pm 0.32$ & $1.29 \pm 0.07$ \\
$10^{2} \mathcal{B}_{\Sigma^{+} \pi^{0}}$ & $1.26 \pm 0.06$ & 2.24 & $1.23 \pm 0.17$ & $1.24 \pm 0.10$ \\
$10^{2} \mathcal{B}_{\Sigma^{+} \eta}$ & $0.29 \pm 0.12$ & 0.74 & $0.47 \pm 0.22$ & $0.41 \pm 0.20$ \\
$10^{2} \mathcal{B}_{\Sigma^{+} \eta^{\prime}}$ & $1.44 \pm 0.56$ & & $0.93 \pm 0.28$ & $1.34 \pm 0.57$ \\
$10^{2} \mathcal{B}_{\Xi^{0} K^{+}}$ & $0.57 \pm 0.09$ & 0.73 & $0.59 \pm 0.17$ & $0.59 \pm 0.09$ \\
$10^{2} \mathcal{B}_{p \bar{K}^{0}}$ & $3.14 \pm 0.15$ & 2.11 & $3.14 \pm 1.00$ & $3.16 \pm 0.16$ \\
$10^{2} \mathcal{B}_{\Lambda^{0} \pi^{+}}$ & $1.27 \pm 0.07$ & 1.30 & $1.32 \pm 0.34$ & $1.30 \pm 0.07$ \\
\hline $10^{4} \mathcal{B}_{\Sigma^{+} K^{0}}$ & $10.5 \pm 1.4$ & 14.4 & $19.1 \pm 4.8$ & \\
$10^{4} \mathcal{B}_{\Sigma^{0} K^{+}}$ & $5.2 \pm 0.7$ & 7.2 & $5.5 \pm 1.6$ & $5.2 \pm 0.8$ \\
$10^{4} \mathcal{B}_{p \pi^{0}}$ & $1.1_{-1.1}^{+1.3}$ & 1.3 & $0.8_{-0.8}^{+0.9}$ & $0.8 \pm 1.4$ \\
$10^{4} \mathcal{B}_{p \eta}$ & $11.2 \pm 2.8$ & 12.8 & $11.4 \pm 3.5$ & $12.4 \pm 3.0$ \\
$10^{4} \mathcal{B}_{p \eta^{\prime}}$ & $24.5 \pm 14.6$ & & $7.1 \pm 1.4$ & \\
$10^{4} \mathcal{B}_{n \pi^{+}}$ & $7.6 \pm 1.1$ & 0.9 & $7.7 \pm 2.0$ & \\
$10^{4} \mathcal{B}_{\Lambda^{0} K^{+}}$ & $6.6 \pm 0.9$ & 10.7 & $5.9 \pm 1.7$ & $6.1 \pm 1.2$ \\
\hline $10^{5} \mathcal{B}_{p K^{0}}$ & $1.2_{-1.2}^{+1.4}$ & & $3.7 \pm 1.1$ & \\
$10^{5} \mathcal{B}_{n K^{+}}$ & $0.4 \pm 0.2$ & & $1.4 \pm 0.5$ & \\
\hline
\end{tabular}

Table 3. The numerical results of the $\mathbf{B}_{c} \rightarrow \mathbf{B} M$ decays with $\mathcal{B}_{\mathbf{B} M} \equiv \mathcal{B}\left(\mathbf{B}_{c} \rightarrow \mathbf{B} M\right)$, in comparison with the results from the $\mathrm{SU}(3)_{f}$ symmetry $[31,35]$ and the calculation with the pole model, current algebra and MIT bag model [32,35]. The data of $\mathcal{B}\left(\Xi_{c}^{0} \rightarrow \Xi^{-} K^{+}, \Lambda^{0} \bar{K}^{0}\right)$ are extracted with $R_{1,2}\left(\Xi_{c}^{0}\right)$, respectively.

where $\overline{\mathcal{A}}_{K}$ is reduced as $\overline{\mathcal{A}}_{K}=V_{c s} V_{u s} T$ with $V_{c s} V_{u s}=-V_{c d} V_{u d}$, resulting in $\mathcal{R}_{1}\left(\Xi_{c}^{0}\right) \simeq$ $\left(1+E_{\mathbf{B}} / T\right)^{2} s_{c}^{2}$ with the value of $(0.54 \pm 0.04) s_{c}^{2}$ to agree with the data. Indeed, the effects of the $\mathrm{SU}(3)_{f}$ symmetry breaking can give rise to the new parameters added to $\Xi_{c}^{0} \rightarrow \Xi^{-} K^{+}$, instead of $\Xi_{c}^{0} \rightarrow \Xi^{-} \pi^{+}$, such that $\mathcal{R}_{1}\left(\Xi_{c}^{0}\right)$ can be fit [25]. Here, our interpretation for $\mathcal{R}_{1}\left(\Xi_{c}^{0}\right)$ relies on the additional diagram in figure 2 without invoking the $\mathrm{SU}(3)_{f}$ symmetry breaking.

By relating the topological diagrams in figure 1 to the symmetry properties of the baryon wave functions, such as the (anti-)symmetric quark ordering of $\Sigma^{0}\left(\Lambda^{0}\right) \sim(u d \pm d u) s$ or the irreducible forms in the $\mathrm{SU}(3)_{f}$ and $\mathrm{SU}(2)$ spin symmetries, one can derive the more restrict parameterization of the topological amplitudes [33,34]. This leads to $\mathcal{R}_{1}\left(\Xi_{c}^{0}\right) \simeq$ $1.0 s_{c}^{2}$ inconsistent with the data. Moreover, the $T$ term, which contributes to $\Lambda_{c}^{+} \rightarrow \Sigma^{0} M^{+}$ with $M^{+}=\left(\pi^{+}, K^{+}\right)$in table 1 , becomes forbidden in refs. [33, 34]. We hence turn off 

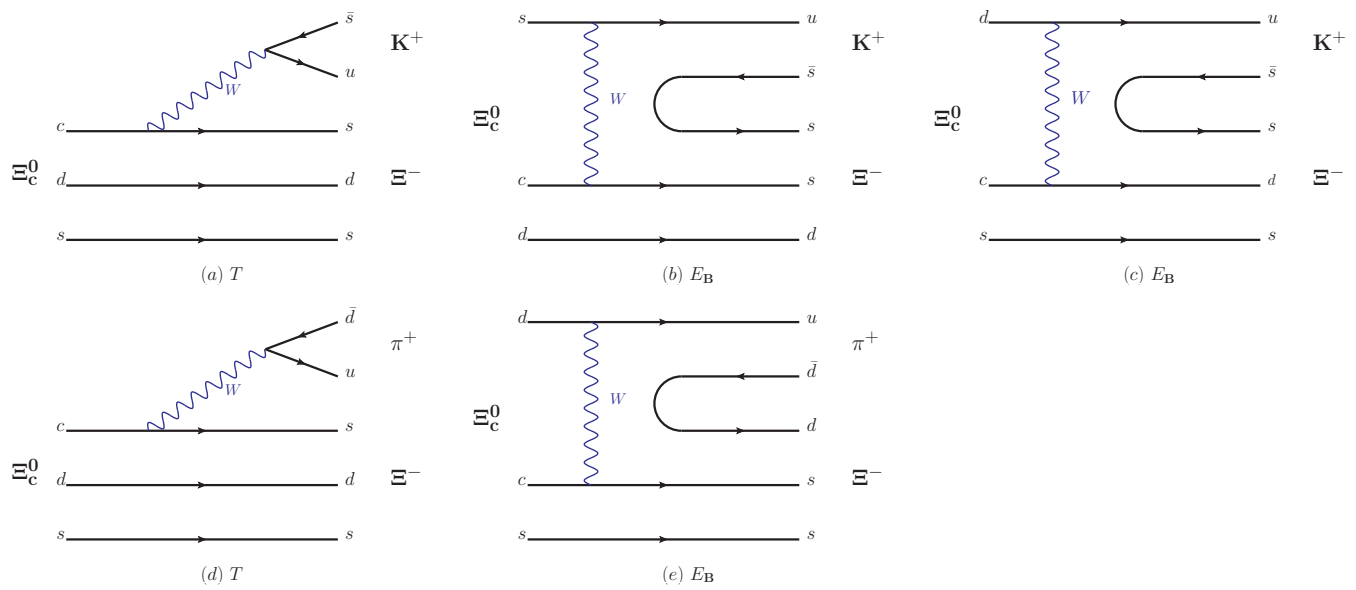

Figure 2. Decay processes (a,b,c) and (d,e) for $\Xi_{c}^{0} \rightarrow \Xi^{-} K^{+}$and $\Xi_{c}^{0} \rightarrow \Xi^{-} \pi^{+}$, respectively.

the $T$ term in $\Lambda_{c}^{+} \rightarrow \Sigma^{0} M^{+}$as a test fit, and obtain $\chi^{2} \sim 30$. Clearly, the more restrict representations cannot explain the data well. Without considering the symmetry properties of the baryon wave functions, our topological amplitudes present the most general forms, which are able to receive the short and long-distance contributions both. The long-distance effect has been proposed in the pole model to contribute to the $W$-exchange process [32, 35]. In the $\Xi_{c}^{0} \rightarrow \Xi^{-} M^{+}$decay, $\Xi_{c}^{0}$ transforms as the $\Lambda^{0}\left(\Sigma^{0}\right)$ pole followed by the strong decay $\Lambda^{0}\left(\Sigma^{0}\right) \rightarrow \Xi^{-} M^{+}$, which contributes to figures $2 \mathrm{~b}$ and c; nonetheless, the latter diagram is forbidden with the more restrict representations. Another example comes from the rescattering effect. With $\Lambda^{*}$ denoting the higher-wave $\Lambda$ state, the $\Lambda_{c}^{+} \rightarrow \Lambda^{*} \rho^{+}$decay has a $T$ amplitude $\left(T^{*}\right)$. Through the $\pi^{0}$ exchange, $\Lambda^{*} \rho^{+}$rescatter into $\Sigma^{0} \pi^{+}$, such that $T^{*}$ contributes to $T$ in $\Lambda_{c}^{+} \rightarrow \Sigma^{0} \pi^{+}$. In fact, the symmetry properties of the meson wave functions are not involved in the $D \rightarrow M M$ decays, such that the long-distance contributions have been absorbed into the topological amplitudes [39, 40, 42, 51].

In table 3 , the $\mathrm{SU}(3)_{f}$ parameters and topological amplitudes that respect the $\mathrm{SU}(3)_{f}$ symmetry are found to explain the data of $\mathcal{B}\left(\Lambda_{c}^{+} \rightarrow \mathbf{B} M\right)$ well, indicating that the $\mathrm{SU}(3)_{f}$ symmetry in $\Lambda_{c}^{+} \rightarrow \mathbf{B} M$ has no sizeable broken effects. In the $D \rightarrow K \bar{K}$ decays, the $W$ exchange processes with $V_{c s} V_{u s}$ and $V_{c d} V_{u d}$ are parameterized as $E^{(d)}$ and $E^{(s)}$, respectively. One needs the sizeable broken effect of $\left|E^{(s)}\right|>\left|E^{(d)}\right|$ to explain $\mathcal{B}\left(D^{0} \rightarrow K^{+} K^{-}\right) / \mathcal{B}\left(D^{0} \rightarrow\right.$ $\left.\pi^{+} \pi^{-}\right)$and $\mathcal{B}\left(D^{0} \rightarrow K^{0} \bar{K}^{0}\right)$ [39-42]. Likewise, since we can present $\mathcal{A}\left(\Xi_{c}^{0} \rightarrow \Xi^{-} K^{+}\right)$as $\overline{\mathcal{A}}_{K} \propto T+\Delta E_{\mathbf{B}}$ with $\Delta E_{\mathbf{B}} \equiv E_{\mathbf{B}}^{(s)}-E_{\mathbf{B}}^{(d)}$, eq. (4.1), whether $\Delta E_{\mathbf{B}}$ is equal to zero or not can be a test of the broken $\mathrm{SU}(3)_{f}$ symmetry. This requires more accurate measurements from BELLEII and LHCb.

We compare the three theoretical results in table 3, which all agree with the observed $\mathcal{B}\left(\Lambda_{c}^{+} \rightarrow \mathbf{B} M\right)$. However, the $\mathrm{SU}(3)_{f}$ symmetry gives $\mathcal{B}\left(\Xi_{c}^{+} \rightarrow \Xi^{0} \pi^{+}\right)$at least 2 times smaller than the observation $[31,35]$. Moreover, the $\mathrm{SU}(3)_{f}$ symmetry and the computations that involve the factorization, pole model, current algebra and MIT bag model present $\mathcal{B}\left(\Xi_{c}^{0} \rightarrow \Xi^{-} \pi^{+}, \Xi^{-} K^{+}, \Lambda^{0} \bar{K}^{0}\right)$ with large deviations from the data [31, 32, 35]. Since we are able to explain $\mathcal{B}\left(\Xi_{c}^{+} \rightarrow \Xi^{0} \pi^{+}\right)$and $\mathcal{B}\left(\Xi_{c}^{0} \rightarrow \Xi^{-} \pi^{+}, \Xi^{-} K^{+}, \Lambda^{0} \bar{K}^{0}\right)$, the 
topological amplitudes are shown to have the advantage to explain the two-body $\Xi_{c}^{0,+}$ decays. To further investigate the $\Xi_{c}^{0,+} \rightarrow \mathbf{B} M$ decays, we present our predictions in table 3. It is worth noting that $E_{M}$ and $E^{\prime}$ give a constructive (destructive) interference to $\Xi_{c}^{0} \rightarrow \Sigma^{+} K^{-}\left(\pi^{-}\right)$, such that we obtain $\mathcal{B}\left(\Xi_{c}^{0} \rightarrow \Sigma^{+} K^{-}\right)=(22.0 \pm 5.7) \times 10^{-3}$ and $\mathcal{B}\left(\Xi_{c}^{0} \rightarrow \Sigma^{+} \pi^{-}\right)=(2.4 \pm 1.5) \times 10^{-4}$, bigger and smaller than the other predictions, respectively. In addition to $\Lambda_{c}^{+} \rightarrow p \pi^{0}$, there are three singly Cabibbo-suppressed $\Lambda_{c}^{+}$decay modes to be measured. Their branching ratios are predicted as $\mathcal{B}\left(\Lambda_{c}^{+} \rightarrow n \pi^{+}, p \eta^{\prime}, \Sigma^{+} K^{0}\right)=$ $(7.7 \pm 2.0,7.1 \pm 1.4,19.1 \pm 4.8) \times 10^{-4}$, where $\mathcal{B}\left(\Lambda_{c}^{+} \rightarrow n \pi^{+}\right)$is consistent with the value from the $\mathrm{SU}(3)_{f}$ symmetry, whereas $\mathcal{B}\left(\Lambda_{c}^{+} \rightarrow \Sigma^{+} K^{0}\right)$ agrees with the theoretical computation in $[32,35]$.

In summary, we have studied the $\mathbf{B}_{c} \rightarrow \mathbf{B} M$ decays within the framework of the diagrammatic approach that respects the $\mathrm{SU}(3)_{f}$ symmetry. With the extraction of the topological amplitudes, we have explicitly presented the two $W$-exchange effects as $E_{\mathbf{B}}$ and $E^{\prime}$ that contribute to the non-factorizable $\Lambda_{c}^{+} \rightarrow \Xi^{0} K^{+}$decay, together with the relative phases, where $E_{\mathbf{B}}$ gives the main contribution. We have obtained $\mathcal{B}\left(\Lambda_{c}^{+} \rightarrow p \pi^{0}\right)=$ $\left(0.8_{-0.8}^{+0.9}\right) \times 10^{-4}$, which agrees with the experimental upper bound. We have presented that $\mathcal{B}\left(\Xi_{c}^{+} \rightarrow \Xi^{0} \pi^{+}\right)=(9.3 \pm 3.6) \times 10^{-3}, \mathcal{B}\left(\Xi_{c}^{0} \rightarrow \Xi^{-} \pi^{+}, \Lambda^{0} \bar{K}^{0}\right)=(19.3 \pm 2.8,8.3 \pm$ $5.0) \times 10^{-2}$ and $\mathcal{B}\left(\Xi_{c}^{0} \rightarrow \Xi^{-} K^{+}\right)=(5.6 \pm 0.8) \times 10^{-4}$ to agree with the data, whereas the other theoretical results have shown sizeable deviations. We have predicted that $\mathcal{B}\left(\Lambda_{c}^{+} \rightarrow\right.$ $\left.n \pi^{+}, p \eta^{\prime}, \Sigma^{+} K^{0}\right)=(7.7 \pm 2.0,7.1 \pm 1.4,19.1 \pm 4.8) \times 10^{-4}$, in order to be compared with future BESIII, BELLEII and LHCb measurements.

\section{Acknowledgments}

We would like to thank Professor H.Y. Cheng, Professor Fanrong Xu, and Professor C.Q. Geng for useful discussions. We would like to thank Dr. Eduardo Rodrigues for reading the manuscript and giving valuable comments. This work was supported by National Science Foundation of China (11675030) and (11905023).

Open Access. This article is distributed under the terms of the Creative Commons Attribution License (CC-BY 4.0), which permits any use, distribution and reproduction in any medium, provided the original author(s) and source are credited.

\section{References}

[1] BESIII collaboration, Measurements of absolute branching fractions for $\Lambda_{c}^{+} \rightarrow \Xi^{0} K^{+}$and $\Xi(1530)^{0} K^{+}$, Phys. Lett. B 783 (2018) 200 [arXiv: 1803.04299] [InSPIRE].

[2] BESIII collaboration, Measurements of absolute hadronic branching fractions of $\Lambda_{c}^{+}$baryon, Phys. Rev. Lett. 116 (2016) 052001 [arXiv:1511.08380] [INSPIRE].

[3] BESIII collaboration, Evidence for the singly-Cabibbo-suppressed decay $\Lambda_{c}^{+} \rightarrow p \eta$ and search for $\Lambda_{c}^{+} \rightarrow p \pi^{0}$, Phys. Rev. D 95 (2017) 111102 [arXiv: 1702.05279] [INSPIRE].

[4] A. Ali, G. Kramer and C.-D. Lu, Experimental tests of factorization in charmless nonleptonic two-body B decays, Phys. Rev. D 58 (1998) 094009 [hep-ph/9804363] [INSPIRE]. 
[5] C.Q. Geng, Y.K. Hsiao and J.N. Ng, Direct CP-violation in $B^{ \pm} \rightarrow p \bar{p} K(*)^{ \pm}$, Phys. Rev. Lett. 98 (2007) 011801 [hep-ph/0608328] [INSPIRE].

[6] Y.K. Hsiao and C.Q. Geng, Direct CP-violation in $\Lambda_{b}$ decays, Phys. Rev. D 91 (2015) 116007 [arXiv: 1412.1899] [INSPIRE].

[7] Y.K. Hsiao, Y. Yao and C.Q. Geng, Charmless two-body anti-triplet b-baryon decays, Phys. Rev. D 95 (2017) 093001 [arXiv: 1702.05263] [InSPIRE].

[8] J.G. Körner and M. Krämer, Exclusive nonleptonic charm baryon decays, Z. Phys. C 55 (1992) 659 [INSPIRE].

[9] P. Zenczykowski, Quark and pole models of nonleptonic decays of charmed baryons, Phys. Rev. D 50 (1994) 402 [hep-ph/9309265] [INSPIRE].

[10] M.A. Ivanov, J.G. Körner, V.E. Lyubovitskij and A.G. Rusetsky, Exclusive nonleptonic decays of bottom and charm baryons in a relativistic three quark model: evaluation of nonfactorizing diagrams, Phys. Rev. D 57 (1998) 5632 [hep-ph/9709372] [INSPIRE].

[11] Q.P. Xu and A.N. Kamal, Cabibbo favored nonleptonic decays of charmed baryons, Phys. Rev. D 46 (1992) 270 [INSPIRE].

[12] K.K. Sharma and R.C. Verma, A study of weak mesonic decays of $\Lambda_{c}$ and $\Xi_{c}$ baryons on the basis of HQET results, Eur. Phys. J. C 7 (1999) 217 [hep-ph/9803302] [INSPIRE].

[13] X.G. He et al., The CP-violating phase $\gamma$ from global fit of rare charmless hadronic $B$ decays, Phys. Rev. D 64 (2001) 034002 [hep-ph/0011337] [InSPIRE].

[14] H.-K. Fu, X.-G. He and Y.-K. Hsiao, $B \rightarrow \eta\left(\eta^{\prime}\right) K(\pi)$ in the standard model with flavor symmetry, Phys. Rev. D 69 (2004) 074002 [hep-ph/0304242] [INSPIRE].

[15] Y.-K. Hsiao, C.-F. Chang and X.-G. He, A global SU(3)/U(3) flavor symmetry analysis for $B \rightarrow$ PP with $\eta-\eta^{\prime}$ mixing, Phys. Rev. D 93 (2016) 114002 [arXiv:1512.09223] [InSPIRE].

[16] X.-G. He and G.-N. Li, Predictive CP violating relations for charmless two-body decays of beauty baryons $\Xi_{b}^{-, 0}$ and $\Lambda_{b}^{0}$ with flavor $\mathrm{SU}(3)$ symmetry, Phys. Lett. B 750 (2015) 82 [arXiv: 1501.00646] [INSPIRE].

[17] M. He, X.-G. He and G.-N. Li, CP-violating polarization asymmetry in charmless two-body decays of beauty baryons, Phys. Rev. D 92 (2015) 036010 [arXiv:1507.07990] [INSPIRE].

[18] M.J. Savage and R.P. Springer, SU(3) predictions for charmed baryon decays, Phys. Rev. D 42 (1990) 1527 [INSPIRE].

[19] M.J. Savage, SU(3) violations in the nonleptonic decay of charmed hadrons, Phys. Lett. B 257 (1991) 414 [inSPIRE].

[20] G. Altarelli, N. Cabibbo and L. Maiani, Weak nonleptonic decays of charmed hadrons, Phys. Lett. B 57 (1975) 277.

[21] C.-D. Lü, W. Wang and F.-S. Yu, Test flavor SU(3) symmetry in exclusive $\Lambda_{c}$ decays, Phys. Rev. D 93 (2016) 056008 [arXiv: 1601.04241] [INSPIRE].

[22] C.Q. Geng, Y.K. Hsiao, Y.-H. Lin and L.-L. Liu, Non-leptonic two-body weak decays of $\Lambda_{c}(2286)$, Phys. Lett. B 776 (2018) 265 [arXiv:1708.02460] [INSPIRE].

[23] C.Q. Geng, Y.K. Hsiao, C.-W. Liu and T.-H. Tsai, Antitriplet charmed baryon decays with SU(3) flavor symmetry, Phys. Rev. D 97 (2018) 073006 [arXiv: 1801.03276] [INSPIRE]. 
[24] C.Q. Geng, Y.K. Hsiao, C.-W. Liu and T.-H. Tsai, Charmed baryon weak decays with $\mathrm{Su}(3)$ flavor symmetry, JHEP 11 (2017) 147 [arXiv:1709.00808] [INSPIRE].

[25] C.Q. Geng, Y.K. Hsiao, C.-W. Liu and T.-H. Tsai, SU(3) symmetry breaking in charmed baryon decays, Eur. Phys. J. C 78 (2018) 593 [arXiv: 1804.01666] [INSPIRE].

[26] W. Wang, Z.-P. Xing and J. Xu, Weak decays of doubly heavy baryons: SU(3) analysis, Eur. Phys. J. C 77 (2017) 800 [arXiv:1707.06570] [INSPIRE].

[27] D. Wang, P.-F. Guo, W.-H. Long and F.-S. Yu, $K_{S}^{0}-K_{L}^{0}$ asymmetries and CP-violation in charmed baryon decays into neutral kaons, JHEP 03 (2018) 066 [arXiv:1709.09873] [INSPIRE].

[28] C.Q. Geng, Y.K. Hsiao, C.-W. Liu and T.-H. Tsai, Three-body charmed baryon Decays with SU(3) flavor symmetry, Phys. Rev. D 99 (2019) 073003 [arXiv: 1810.01079] [InSPIRE].

[29] Y.K. Hsiao, Y. Yao and H.J. Zhao, Two-body charmed baryon decays involving vector meson with SU(3) flavor symmetry, Phys. Lett. B 792 (2019) 35 [arXiv:1902.08783] [INSPIRE].

[30] C.-Q. Geng, C.-W. Liu and T.-H. Tsai, Singly Cabibbo suppressed decays of $\Lambda_{c}^{+}$with $\mathrm{SU}(3)$ flavor symmetry, Phys. Lett. B 790 (2019) 225 [arXiv:1812.08508] [INSPIRE].

[31] C.Q. Geng, C.-W. Liu and T.-H. Tsai, Asymmetries of anti-triplet charmed baryon decays, Phys. Lett. B 794 (2019) 19 [arXiv: 1902.06189] [INSPIRE].

[32] H.-Y. Cheng, X.-W. Kang and F. Xu, Singly Cabibbo-suppressed hadronic decays of $\Lambda_{c}^{+}$, Phys. Rev. D 97 (2018) 074028 [arXiv:1801.08625] [INSPIRE].

[33] Y. Kohara, Quark diagram analysis of charmed baryon decays, Phys. Rev. D 44 (1991) 2799 [INSPIRE].

[34] L.-L. Chau, H.-Y. Cheng and B. Tseng, Analysis of two-body decays of charmed baryons using the quark diagram scheme, Phys. Rev. D 54 (1996) 2132 [hep-ph/9508382] [INSPIRE].

[35] J. Zou, F. Xu, G. Meng and H.-Y. Cheng, Two-body hadronic weak decays of antitriplet charmed baryons, Phys. Rev. D 101 (2020) 014011 [arXiv:1910.13626] [INSPIRE].

[36] A.K. Leibovich, Z. Ligeti, I.W. Stewart and M.B. Wise, Predictions for nonleptonic $\Lambda_{b}$ and $\Theta_{b}$ decays, Phys. Lett. B 586 (2004) 337 [hep-ph/0312319] [INSPIRE].

[37] D. Pirtskhalava and P. Uttayarat, CP violation and flavor $\mathrm{SU}(3)$ breaking in D-meson decays, Phys. Lett. B 712 (2012) 81 [arXiv:1112.5451] [inSPIRE].

[38] Y. Grossman and D.J. Robinson, SU(3) sum rules for charm decay, JHEP 04 (2013) 067 [arXiv:1211.3361] [INSPIRE].

[39] H.-Y. Cheng and C.-W. Chiang, SU(3) symmetry breaking and CP-violation in $D \rightarrow P P$ decays, Phys. Rev. D 86 (2012) 014014 [arXiv:1205.0580] [InSPIRE].

[40] H.-n. Li, C.-D. Lu and F.-S. Yu, Branching ratios and direct CP asymmetries in $D \rightarrow P P$ decays, Phys. Rev. D 86 (2012) 036012 [arXiv: 1203.3120] [INSPIRE].

[41] H.-n. Li, C.-D. Lü, Q. Qin and F.-S. Yu, Branching ratios and direct CP asymmetries in $D \rightarrow P V$ decays, Phys. Rev. D 89 (2014) 054006 [arXiv:1305.7021] [InSPIRE].

[42] H.-Y. Cheng and C.-W. Chiang, Revisiting CP-violation in D $\rightarrow P P$ and VP decays, Phys. Rev. D 100 (2019) 093002 [arXiv: 1909.03063] [INSPIRE].

[43] Particle Data Group collaboration, Review of particle physics, Phys. Rev. D 98 (2018) 030001 [INSPIRE]. 
[44] A.J. Buras, Weak Hamiltonian, CP-violation and rare decays, hep-ph/9806471 [INSPIRE].

[45] T. Feldmann, P. Kroll and B. Stech, Mixing and decay constants of pseudoscalar mesons: The Sequel, Phys. Lett. B 449 (1999) 339 [hep-ph/9812269] [InSPIRE].

[46] BELLE collaboration, First measurements of absolute branching fractions of the $\Xi_{c}^{0}$ baryon at Belle, Phys. Rev. Lett. 122 (2019) 082001 [arXiv:1811.09738] [INSPIRE].

[47] BESIII collaboration, Evidence for the decays of $\Lambda_{c}^{+} \rightarrow \Sigma^{+} \eta$ and $\Sigma^{+} \eta^{\prime}$, Chin. Phys. C 43 (2019) 083002 [arXiv:1811.08028] [INSPIRE].

[48] P. Li, private communication.

[49] BELLE collaboration, First measurements of absolute branching fractions of the $\Xi_{c}^{+}$baryon at Belle, Phys. Rev. D 100 (2019) 031101 [arXiv:1904.12093] [inSPIRE].

[50] X.-G. He, Y.-J. Shi and W. Wang, Unification of flavor SU(3) analyses of heavy hadron weak decays, arXiv: 1811.03480 [INSPIRE].

[51] Y.K. Hsiao, Y. Yu and B.-C. Ke, Evidence for $a_{0}(980)$ as tetraquark from the triangle rescattering $D_{s}^{+} \rightarrow \pi^{+} \pi^{0} \eta$ decay, arXiv:1909.07327 [INSPIRE]. 